\title{
Transmuted extended Lomax distribution with some tractability properties and applications
}

\author{
Faten A. Momenkhan ${ }^{\mathrm{a}, \mathrm{b}}$ \\ ${ }^{a}$ Department of Statistics, Faculty of Science, University of Jeddah, Jeddah, Saudi Arabia. \\ ${ }^{\mathrm{b}}$ Department of Statistics, Faculty of Science-Al Faisaliah, King Abdulaziz University, Jeddah, Saudi Arabia. \\ ${ }^{1}$ E-mail: fatenmomen@hotmail.com
}

\begin{abstract}
Extending or generalizing original distributions create new distributions with some tractability properties and with more flexibility in modeling data. In this paper, the extended Lomax distribution introduced by Ghitany et al. [1] is further extended in a larger family by introducing an additional parameter. We provide a comprehensive description of the mathematical properties of the subject distribution along with its reliability behavior. The problem of the parameter estimation for the proposed distribution is considered based on the maximum likelihood approach. Finally, the usefulness of the transmuted distribution for modeling reliability data is illustrated using a simulation study and a real data set.
\end{abstract}

Keywords: Extended Lomax distribution; hazard rate function; reliability function; parameter estimation.

\section{Introduction}

The Lomax distribution has been extensively used for reliability modeling and life testing see e.g. Balkema and de Haan [2]. It also has been used as an alternative to the exponential distribution when the data are heavy tailed, see Bryson [3]. It has applications in economics, actuarial modeling, queuing problems and biological sciences, for details we refer to Johnson et al. [4].

Definition 1. A random variable $X$ is said to have Lomax distribution, abbreviated as $X \sim L(\alpha, \beta)$, if it has the probability density function (pdf)

$$
f(x ; \alpha, \beta)=\alpha \beta(1+\beta x)^{-(\alpha+1)}, x \geq 0 ; \alpha, \beta>0 .
$$

Here $\alpha$ and $\beta$ are the shape and scale parameters, respectively.

The survival function (sf) associated to Eq. (1) is given by

$$
\bar{F}_{L}(x ; \alpha, \beta)=(1+\beta x)^{-\alpha}, \quad x \geq 0 ; \alpha, \beta>0 .
$$

We are not focusing our attention on the Lomax distribution but further probabilistic properties of this distribution can be found in many sources, for example, in Arnold [5]. Reference [1] presented the extended Lomax distribution by adding a new shape parameter using the idea of Marshall and Olkin [6].

Definition 2. A random variable $X$ possessing the Marshall-Olkin extended Lomax distribution, denoted by $X \sim E L(\alpha, \beta, \gamma)$, if its cumulative distribution function (cdf) is

$$
\bar{F}_{E L}(x ; \alpha, \beta, \gamma)=\frac{\alpha}{(1+\beta x)^{\gamma}-\bar{\alpha}}
$$

where the parameters $\alpha, \beta, \gamma>0$ and $\bar{\alpha}=1-\alpha$.

Reference [1] studied the shapes of the density function associated with Eq. (3) and derived the moments and quartiles. They also discussed the shapes of the hazard rate function and gave limiting distributions of sample extremes.

The main purpose of this paper is to introduce and study a three-parameter lifetime distribution by transmuted the extended Lomax distribution. The proposed model is observed to be a heavy tailed distribution and has a decreasing or upside-down bathtub shaped failure 
rates depending on its parameters. Heavy tailed distributions have many applications and can be used to model, for example, job sizes, computer file sizes stored on a server and transmission rates of files. A few standard lifetime distributions with the property 'upside-down bathtub shaped' are found in the literature, so it is of interest to have a distribution can model practical applications, for example large sample of washing machines, see Schabe [7].

Definition 3. A random variable $X$ is said to have transmuted distribution if its $c d f$ is given by

$$
F(x)=(1+\lambda) G(x)-\lambda[G(x)]^{2}, \quad-1 \leq \lambda \leq 1,
$$

where $G(x)$ is the cdf of the base distribution.

Observe that at $\lambda=0$ we have the distribution of the base random variable. Aryal and Tsokos [8] studied the transmuted Gumbel distribution and it has been observed that transmuted Gumbel distribution can be used to model climate data. Aryal and Tsokos ([8] and [9]) studied the transmuted extreme value distribution and Weibull distribution. The authors provided the mathematical characterization of the mentioned distributions and used them to analyze real data sets. Also, Aryal [10] studied the transmuted log logistic distribution and discussed some properties of this family.

\section{Model Formulation}

Substitution (3) into (4) yields the following:

$$
\begin{aligned}
F(x ; \alpha, \beta, \lambda, \gamma)= & (1+\lambda)\left\{1-\frac{\alpha}{(1+\beta x)^{\gamma}-\bar{\alpha}}\right\} \\
& -\lambda\left\{1-\frac{\alpha}{(1+\beta x)^{\gamma}-\bar{\alpha}}\right\}^{2}, x>0 .
\end{aligned}
$$

We study the distributional properties of the TEL distribution. In particular, the shapes of the density function, the shapes of the hazard function, moments. As a consequences, the density of the $r$ th order statistics, and the mean and median deviations of the TEL distribution can be derived and studied in detail.

\subsection{Shapes of pdf and hrf}

A random variable $X$ is said to have a transmuted extended Lomax distribution with parameters $\alpha, \beta, \lambda$ and $\gamma$, abbreviated as, $X \sim$ $\operatorname{TEL}(\alpha, \beta, \lambda, \gamma)$, if its pdf is given by

$$
\begin{aligned}
f(x ; \alpha, \beta, \lambda, \gamma)= & \frac{\alpha \beta \lambda(1+\beta x)^{\gamma-1}}{(1+\beta x)^{\gamma}-\bar{\alpha}}\{1+\lambda-2 \lambda \\
& \left.\times\left(1-\frac{\alpha}{(1+\beta x)^{\gamma}-\bar{\alpha}}\right)\right\}, x>0,
\end{aligned}
$$

for $\alpha, \beta, \lambda, \gamma>0$. The limit of the density given in (6) as $x \rightarrow \infty$ is 0 and the limit as $x \rightarrow 0$ is $\beta \lambda(1+\lambda)$. Now, we give simple conditions under which the pdf is decreasing or unimodal.

The pdf, $f(x)$ of $X \sim T E L(\alpha, \beta, \lambda, \gamma)$, is decreasing (unimodal) depending on the values of the parameters. We can write the pdf $f$ in terms of the pdf $f_{E L}$ as follows

$$
f(x)=(1+\lambda) f_{E L}(x)-2 \lambda f_{E L}(x) F_{E L}(x)
$$

This enables us to find the critical points of the density function $f$ which are the roots of the equation

$$
\frac{f_{E L}^{\prime}(x)}{f_{E L}(x)}-\frac{2 \lambda f_{E L}(x)}{1+\lambda-2 \lambda F_{E L}(x)}=0
$$

There may be more than one root to (7). To study the shape of the density, let $\delta(x)$ be the second derivative of the log of the density function i.e. $\delta(x)=\left(d^{2} / d x^{2}\right)(\log f(x))$. Then, we have

$$
\begin{aligned}
\delta(x) & =\frac{f_{E L}^{\prime \prime}(x) f_{E L}(x)-\left[f_{E L}^{\prime}(x)\right]^{2}}{\left[f_{E L}(x)\right]^{2}} \\
& =-\frac{2 \lambda\left[(1+\lambda) f_{E L}^{\prime}(x)-2 \lambda f_{E L}^{\prime}(x) F_{E L}(x)+2 \lambda\left[f_{E L}(x)\right]^{2}\right]}{\left[1+\lambda-2 \lambda F_{E L}(x)\right]^{2}}
\end{aligned}
$$

If $x=x_{0}$ is a root of $\delta(x)$, then it corresponds to a local minimum if $\delta(x)<0$ for all $x<x_{0}$ and $\delta(x)>0$ for all $x>x_{0}$. it corresponds to a local maximum if $\delta(x)>0$ for all $x<x_{0}$ and $\delta(x)<0$ for all $x>x_{0}$. Fig. 1 shows different possible shapes of the pdf.

The hazard rate function (hrf) of $X \sim T E L(\alpha, \beta, \lambda, \gamma)$, is given by

After some transformation the hrf is given by

$$
h(x)=\frac{\left[1+\lambda-2 \lambda F_{E L}(x)\right] f_{E L}(x)}{\left(1-F_{E L}(x)\right)\left[1-\lambda F_{E L}\right]}
$$

$$
h(x)=\frac{\beta \gamma\left(1+\lambda-2 \lambda\left(1-\frac{\alpha}{(1+\beta x)^{\gamma-\bar{\alpha}}}\right)\right)}{\left.(1+\beta x)-\bar{\alpha}(1+\beta x)^{1-\gamma}-\lambda\left((1+\beta x)^{\gamma}-1\right)\right)}
$$

It can be noted from Eq. (9) that the function $h(x) / h_{E L}(x)$ is decreasing in $x$ for all $\lambda \geq 0$ and it is increasing in $x$ for all $\lambda<0$. Moreover, the hazard rate function of $X \sim T E L(\alpha, \beta, \lambda, \gamma)$, is smaller than that of the extended Lomax distribution i.e. $h(x)<h_{E L}(x)$ for $\lambda<0$. Fig. 2 shows different shapes of the hrf. 


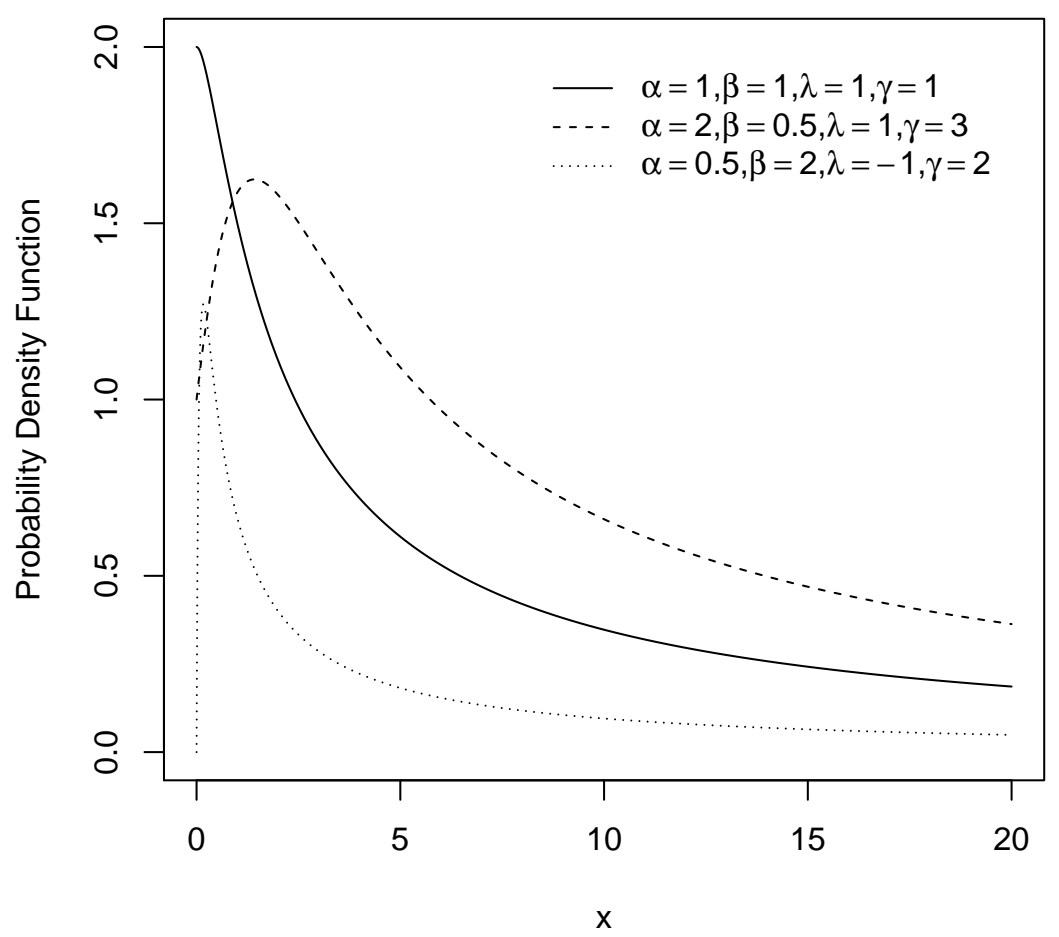

Figure 1: Plot of the density function for different values of the parameters.

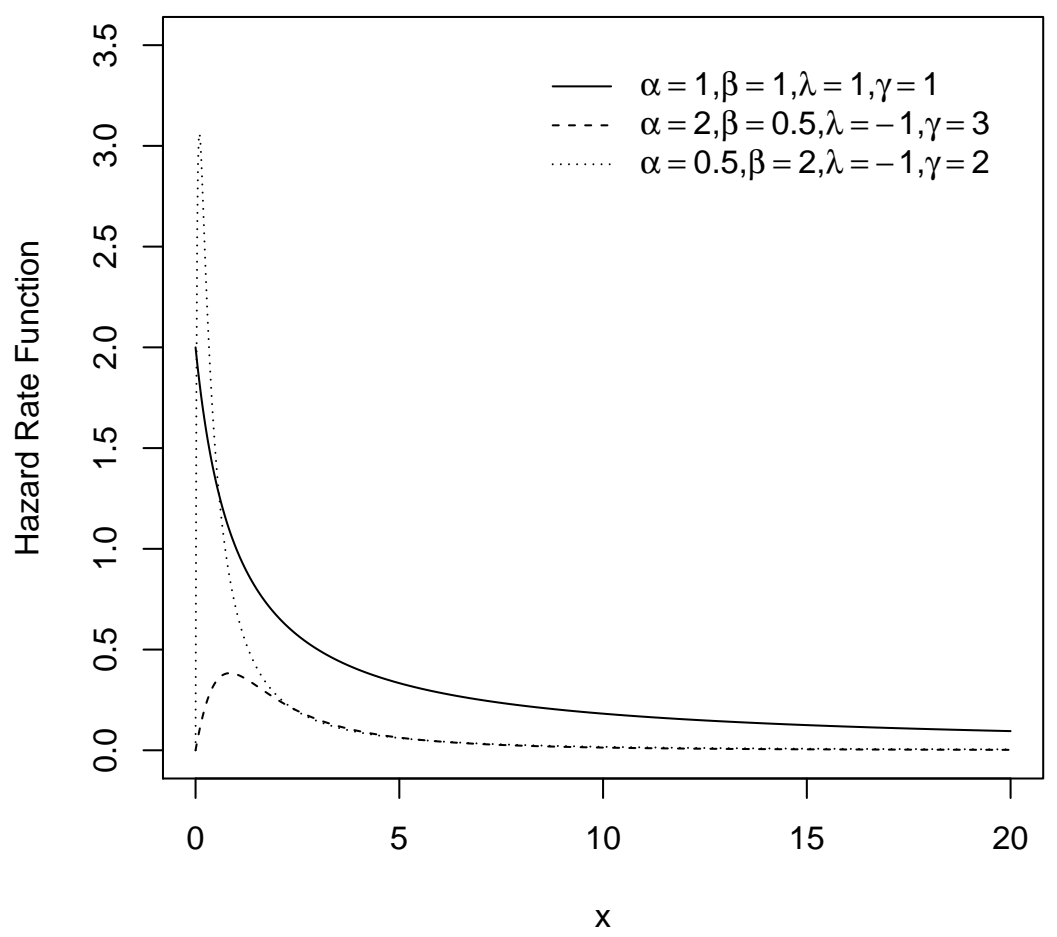

Figure 2: Plot of the hazard rate function for different values of the parameters. 
Table 1: The quartile values of the TEL distribution for different values of $\alpha, \beta, \lambda$ and $\gamma$.

\begin{tabular}{|c|c|c|c|c|c|c|}
\hline \multirow[b]{2}{*}{$\alpha$} & \multirow[b]{2}{*}{ Quartiles } & \multicolumn{4}{|c|}{$\beta=1$ and $\lambda=1$} & \multirow[b]{2}{*}{$\gamma=2.5$} \\
\hline & & $\gamma=0.5$ & $\gamma=1.0$ & $\gamma=1.5$ & $\gamma=2.0$ & \\
\hline \multirow{4}{*}{0.5} & $Q_{1}$ & 0.1642 & 0.0790 & 0.0520 & 0.0393 & 0.0309 \\
\hline & $Q_{2}$ & 0.4663 & 0.2109 & 0.1361 & 0.1035 & 0.0796 \\
\hline & $Q_{3}$ & 1.2374 & 0.4958 & 0.3079 & 0.2215 & 0.1748 \\
\hline & $Q_{1}$ & 0.3409 & 0.1580 & 0.1027 & 0.0771 & 0.0604 \\
\hline \multirow[t]{3}{*}{1} & $Q_{2}$ & 1.0216 & 0.4218 & 0.2644 & 0.1982 & 0.1512 \\
\hline & $Q_{3}$ & 2.9664 & 0.9916 & 0.5829 & 0.4085 & 0.3173 \\
\hline & $Q_{1}$ & 0.5301 & 0.2370 & 0.1523 & 0.1137 & 0.0888 \\
\hline \multirow[t]{3}{*}{1.5} & $Q_{2}$ & 1.6658 & 0.6327 & 0.3866 & 0.2859 & 0.2166 \\
\hline & $Q_{3}$ & 5.1870 & 1.4874 & 0.8358 & 0.5735 & 0.4398 \\
\hline & $Q_{1}$ & 0.7318 & 0.3160 & 0.2009 & 0.1491 & 0.1161 \\
\hline \multirow[t]{4}{*}{2} & $Q_{2}$ & 2.3990 & 0.8436 & 0.5035 & 0.3679 & 0.2772 \\
\hline & $Q_{3}$ & 7.8993 & 1.9832 & 1.0723 & 0.7227 & 0.5484 \\
\hline & \multicolumn{6}{|c|}{$\beta=1$ and $\lambda=-1$} \\
\hline & $Q_{1}$ & -0.4100 & -0.2319 & -0.1613 & -0.1235 & -0.1001 \\
\hline \multirow[t]{3}{*}{0.5} & $Q_{2}$ & -0.3723 & -0.2078 & -0.1438 & -0.1106 & -0.0889 \\
\hline & $Q_{3}$ & -0.3071 & -0.1676 & -0.1151 & -0.0880 & -0.0708 \\
\hline & $Q_{1}$ & -0.7124 & -0.4637 & -0.3399 & -0.2675 & -0.2206 \\
\hline \multirow[t]{3}{*}{1} & $Q_{2}$ & -0.6584 & -0.4155 & -0.3009 & -0.2370 & -0.1933 \\
\hline & $Q_{3}$ & -0.5581 & -0.3352 & -0.2383 & -0.1854 & -0.1507 \\
\hline & $Q_{1}$ & -0.9074 & -0.6956 & -0.5475 & -0.4479 & -0.3786 \\
\hline \multirow[t]{3}{*}{1.5} & $Q_{2}$ & -0.8581 & -0.6233 & -0.4784 & -0.3890 & -0.3233 \\
\hline & $Q_{3}$ & -0.7528 & -0.5028 & -0.3724 & -0.2962 & -0.2439 \\
\hline & $Q_{1}$ & -0.9947 & -0.9275 & -0.8261 & -0.7295 & -0.6499 \\
\hline \multirow[t]{2}{*}{2} & $Q_{2}$ & -0.9714 & -0.8310 & -0.6943 & -0.5944 & -0.5089 \\
\hline & $Q_{3}$ & -0.8914 & -0.6704 & -0.5229 & -0.4281 & -0.3585 \\
\hline
\end{tabular}

\subsection{Quantile function}

Let $X$ denote a random variable with the pdf given by (6). The quantile denoted by $Q\left(u^{*}\right)$

$$
Q\left(u^{*}\right)=\inf \left\{x \in: F(x) \geq u^{*}\right\},
$$

where $0<u^{*}<1$. By inverting the distribution function $F$, we can write the following:

$$
Q\left(u^{*}\right)=Q_{E L}\left(\frac{1+\lambda-\sqrt{(1+)^{2}-4 u^{*}}}{2}, \theta\right)
$$

where $\theta=(\alpha, \beta, \gamma)$ and $Q_{E L}$ is the quantile function of the extended Lomax distribution. The first quartile $Q_{1}$, the second quartile or the median $Q_{2}$ and the third quartile $Q_{3}$, can be obtained simply by applying (10). The quartile values of the TEL distribution for different values of the parameters are given in Table 1.

\subsection{Moments}

Through the ordinary moments of a distribution many of its important characteristics and features can be determined. We shall present the moments of a random variable $X$ where $X \sim T E L(\alpha, \beta, \lambda, \gamma)$.

The $r$ th moment of $X$ is given by

$$
E\left[X^{r}\right]=r \int_{0}^{\infty} X^{r-1} \bar{F}(x) d x
$$

where $\bar{F}(x)=\left(1-F_{E L}(x)\right)\left(1-\lambda F_{E L}(x)\right)$ is the survival function of $X$. After some transformations, we arrive at the following:

$$
\begin{aligned}
E\left[X^{r}\right]= & \frac{r}{\beta^{r} \gamma} \sum_{i=0}^{r-1}\left(\begin{array}{c}
r-1 \\
i
\end{array}\right)(-1)^{r-1-i} \int_{1}^{\infty} y^{\frac{i+1}{\gamma}-1}\left\{\frac{\alpha}{y-\bar{\alpha}}+\lambda\right. \\
& \left.\times\left(\frac{\alpha}{y-\bar{\alpha}}-1+\left(1-\frac{\alpha}{y-\bar{\alpha}}\right)^{2}\right)\right\} d y
\end{aligned}
$$

When $\lambda=0$ we immediately get the $r$ th moment of the Marshall-Olkin extended Lomax distribution. An alternative way to express the rmoment can be obtained through the pdf, and after some transformation it is as follows:

$$
E\left[X^{r}\right]=\sum_{k=0}^{r}\left(\begin{array}{l}
r \\
k
\end{array}\right)(-1)^{k} \sum_{m=0}^{\frac{r-k}{\gamma}}\left(\begin{array}{c}
\frac{r-k}{\gamma} \\
m
\end{array}\right) \alpha^{-m} \alpha^{\frac{r-k}{\gamma}-m} \frac{1}{-\frac{r-k}{\gamma}+m+1} .
$$




\section{Estimation}

The method of maximum likelihood is the most frequently used method of parameter estimation (see Casella and Berger [11]). The method's success stems no doubt from its many desirable properties including consistency, asymptotic efficiency, invariance and simply its intuitive appeal. Also, the maximum likelihood estimation (MLEs) can be used to obtain confidence intervals for the model parameters.

In this section, we consider the estimation of the unknown parameters of the TEL distribution from complete samples. Let $x_{1}, x_{2}, \cdots, x_{n}$ be a random sample of size $n$ from $T E L(\alpha, \beta, \lambda, \gamma)$ distribution. The $\log$-likelihood function for $\theta=(\alpha, \beta, \lambda, \gamma)$ is given by

$$
\begin{aligned}
l(\theta)= & n \log (\alpha)+n \log (\beta)+n \log (\lambda) \\
& +(\gamma-1) \sum_{i=1}^{n} \log \left(1+\beta x_{i}\right)-\sum_{i=1}^{n} \log \left[\left(1+\beta x_{i}\right)^{\gamma}-\bar{\alpha}\right] \\
& +\sum_{i=1}^{n} \log \left[1+\lambda-2 \lambda\left(1-\frac{\alpha}{\left(1+\beta x_{i}\right)^{\gamma}-\bar{\alpha}}\right)\right]
\end{aligned}
$$

The maximum likelihood estimators of $\theta$, say $\hat{\theta}$ can be obtained by finding the solutions of a system of equations obtained by letting the first partial derivatives of the log-likelihood equation equal to zero with respect to $\theta$. The system of equations is as follows:

$$
\begin{gathered}
\frac{\partial l}{\partial \alpha}=\frac{n}{\alpha}-\sum_{i=1}^{n} \frac{1}{\left(1+\beta x_{i}\right)^{\gamma}-\bar{\alpha}} \\
\quad+\sum_{i=1}^{n} \frac{2 \lambda\left[\left(1+\beta x_{i}\right)^{\gamma}-1\right]}{\left[\left(1+\beta x_{i}\right)^{\gamma}-\bar{\alpha}\right]^{2}\left[1+\lambda-2 \lambda\left(1-\frac{\alpha}{\left(1+\beta x_{i}\right)^{\gamma-\bar{\alpha}}}\right)\right]} . \\
\frac{\partial l}{\partial \beta}=\frac{n}{\beta}+(\gamma-1) \sum_{i=1}^{n} \frac{x_{i}}{1+\beta x_{i}}-\sum_{i=1}^{n} \frac{\gamma x_{i}\left(1+\beta x_{i}\right)^{\gamma-1}}{\left(1+\beta x_{i}\right)^{\gamma}-\bar{\alpha}} \\
-\sum_{i=1}^{n} \frac{2 \alpha \lambda \gamma x_{i}\left(1+\beta x_{i}\right)^{\gamma-1}}{\left[\left(1+\beta x_{i}\right)^{\gamma}-\bar{\alpha}\right]^{2}\left[1+\lambda-2 \lambda\left(1-\frac{\alpha}{\left(1+\beta x_{i}\right)^{\gamma-\bar{\alpha}}}\right)\right]} . \\
\frac{\partial l}{\partial \lambda}=\frac{n}{\lambda}+\sum_{i=1}^{n} \frac{1-2\left(1-\frac{\alpha}{1+\lambda-2 \lambda\left(1-\frac{\alpha}{\left.\left(1+\beta x_{i}\right)^{\gamma}-\bar{\alpha}\right)}\right)}\right.}{\frac{\partial l}{\partial \gamma}}=\sum_{i=1}^{n} \log \left(1+\beta x_{i}\right)-\sum_{i=1}^{n} \frac{\left(1+\beta x_{i}\right)^{\gamma} \log \left(1+\beta x_{i}\right)}{\left(1+\beta x_{i}\right)^{\gamma}-\bar{\alpha}} \\
-\sum_{i=1}^{n} \frac{2 \alpha \lambda\left(1+\beta x_{i}\right)^{\gamma} \log \left(1+\beta x_{i}\right)}{\left[\left(1+\beta x_{i}\right)^{\gamma}-\bar{\alpha}\right]^{2}\left[1+\lambda-2 \lambda\left(1-\frac{\alpha}{\left(1+\beta x_{i}\right)^{\gamma-\bar{\alpha}}}\right)\right]} .
\end{gathered}
$$

The solutions of the above nonlinear equations are complicated to obtain, therefore an iterative procedure is applied to solve these equations numerically.

\section{An application}

The fit of the TEL distribution to a set of real data is examined using maximum likelihood estimates. The data set is corresponding to remission times (in months) of a random sample of 128 bladder cancer patients. The bladder cancer data were analyzed by Lee and Wang [12] and further discussed in Al-Zahrani and Sagor [13]. The data set was analyzed by Holland et al. [14] and found to be adequately fitted by the Lomax distribution. The proposed TEL distribution is compared with Lomax, extended Lomax and Poisson-Lomax distributions. The model selection is carried out using the Akaike information criterion (AIC), the Bayesian information criterion, the consistent Akaike information criteria (CAIC) and the Hannan-Quinn information criterion (HQIC).

$$
\begin{aligned}
& \mathrm{AIC}=-2 l(\hat{\boldsymbol{\theta}})+2 q \\
& \mathrm{BIC}=-2 l(\hat{\boldsymbol{\theta}})+q \log (n) \\
& \mathrm{HQIC}=-2 l(\hat{\theta})+2 q \log (\log (n)) \\
& \mathrm{CAIC}=-2 l(\hat{\boldsymbol{\theta}})+\frac{2 q n}{n-q-1},
\end{aligned}
$$

where $l(\hat{\theta})$ denotes the log-likelihood function evaluated at the maximum likelihood estimates, $q$ is the number of parameters, and $n$ is the sample size. The model with minimum AIC or (BIC, CAIC and HQIC) value is chosen as the best model to fit the data. From Table 2, we conclude that the TEL distribution is comparable to Lomax, extended Lomax and Poisson-Lomax models.

\section{Conclusion}

A new four-parameter distribution, known as transmuted extended Lomax distribution has been proposed. The mathematical properties of the proposed distribution have been provided. The moments of the distribution and the quantile function have been obtained. The estimation of the involved parameters has been approached by maximum likelihood. Finally, two real data sets were analyzed to show the potential of the distribution. The result indicates that the TEL distribution behave very similarly, and even in some situations provide a better fit than other well-known distributions. 
Table 2: MLEs (standard errors in parentheses) and the measures AIC, BIC, HQIC and CAIC for bladder cancer data.

\begin{tabular}{|c|c|c|c|c|c|c|c|c|}
\hline \multirow[b]{2}{*}{ Models } & \multicolumn{4}{|c|}{ Estimates } & \multicolumn{4}{|c|}{ Measures } \\
\hline & $\hat{\alpha}$ & $\hat{\beta}$ & $\hat{\gamma}$ & $\hat{\lambda}$ & $\mathrm{AIC}$ & $\mathrm{BIC}$ & HQIC & CAIC \\
\hline Lomax & $\begin{array}{l}13.9384 \\
(15.3837)\end{array}$ & $\begin{array}{l}121.0221 \\
(142.6940)\end{array}$ & & & 831.67 & 837.37 & 833.98 & 831.76 \\
\hline EL & $\begin{array}{l}23.7437 \\
(35.8106)\end{array}$ & $\begin{array}{l}2.0487 \\
(2.5891)\end{array}$ & $\begin{array}{l}2.2818 \\
(0.5551)\end{array}$ & & 825.08 & 833.64 & 828.56 & 825.27 \\
\hline PL & $\begin{array}{l}2.8739 \\
(0.8870)\end{array}$ & $\begin{array}{l}8.2719 \\
(4.8804)\end{array}$ & & $\begin{array}{l}3.3513 \\
(1.0303)\end{array}$ & 824.77 & 833.33 & 828.25 & 824.96 \\
\hline EPL & $\begin{array}{l}0.2387 \\
(1.1424)\end{array}$ & $\begin{array}{l}124.3010 \\
(155.3534)\end{array}$ & & $\begin{array}{l}59.8378 \\
(242.1564)\end{array}$ & 833.67 & 842.22 & 837.14 & 833.86 \\
\hline TEL & $\begin{array}{l}25.2058 \\
(1.7506)\end{array}$ & $\begin{array}{l}119.7928 \\
(0.4615)\end{array}$ & $\begin{array}{l}41.7043 \\
(0.1327)\end{array}$ & $\begin{array}{l}0.8045 \\
(0.41807)\end{array}$ & 825.10 & 836.51 & 829.73 & 825.42 \\
\hline
\end{tabular}

\section{Acknowledgement}

The author is grateful to the editor and anonymous referees for their valuable comments which improved the presentation of the paper.

\section{References}

[1] Ghitany, M. E., Al-Awadhi, F. A., \& Alkhalfan, L. A. (2007). Marshall-Olkin extended Lomax distribution and its application to censored data. Communications in Statistics-Theory and Methods, 36(10), 1855-1866.

[2] Balkema, A. A., \& De Haan, L. (1974). Residual life time at great age. The Annals of Probability, $792-804$.

[3] Bryson, M. C. (1974). Heavy-tailed distributions: properties and tests. Technometrics, 16(1), 61-68.

[4] Johnson, N. L., Kotz, S., \& Balakrishnan, N. (1994). Continuous Univariate Distributions, vol. 1.

[5] Arnold, B. C. (1983). Pareto Distributions, International Co-operative Publishing House, Fairland, MD

[6] Marshall, A. W., \& Olkin, I. (1997). A new method for adding a parameter to a family of distributions with application to the exponential and Weibull families. Biometrika, 84(3), 641-652.

[7] Schäbe, H. (2011, September). A spot of an upside down bathtub failure rate lifetime distribution in the wild. In Safety and Reliability (Vol. 31, No. 3, pp. 74-83). Taylor \& Francis.

[8] Aryal, G. R., \& Tsokos, C. P. (2009). On the transmuted extreme value distribution with application. Nonlinear Analysis: Theory, Methods \& Applications, 71(12), e1401-e1407.

[9] Aryal, G. R., \& Tsokos, C. P. (2011). Transmuted Weibull distribution: A generalization of theWeibull probability distribution. European Journal of Pure and Applied Mathematics, 4(2), 89-102.

[10] Aryal, G. R. (2013). Transmuted log-logistic distribution. Journal of Statistics Applications \& Probability, $2(1), 11$.

[11] Casella, G., \& Berger, R. L. (1990). Statistical inference. Wadsworth \& Brooks. Pacic Grove, CA.

[12] Lee, E. T., \& Wang, J. (2003). Statistical methods for survival data analysis (Vol. 476). John Wiley \& Sons.

[13] Al-Zahrani, B., \& Sagor, H. (2014). The Poisson-Lomax distribution. Revista Colombiana de Estadística, 37(1), $225-245$.

[14] Holland, O., Golaup, A., \& Aghvami, A. H. (2006). Traffic characteristics of aggregated module downloads for mobile terminal reconfiguration. IEE Proceedings-Communications, 153(5), 683-690. 